TRANSACTIONS OF THE

AMERICAN MATHEMATICAL SOCIETY

Volume 173, November 1972

\title{
SOME RESULTS ON PARAFREE GROUPS
}

\author{
BY
}

\section{YAEL ROITBERG}

\begin{abstract}
We obtain some theorems concerning parafree groups in certain varieties, which are analogs of corresponding theorems about free groups in these varieties. Our principal results are: (1) A normal subgroup $N$ of a parafree metabelian group $P$ of rank $\geq 2$ such that $N \cdot \gamma_{2} P$ has infinite index in $P$ is not finitely generated unless it is trivial. (2) If $x$ and $y$ are elements of a parafree group $P$ in any variety containing the variety of all metabelian groups which are independent modulo $\gamma_{2} P$, then the commutator $[x, y]$ is not a proper power.
\end{abstract}

Introduction. In this paper, we shall prove analogs, for parafree groups(1) in certain varieties, of some theorems concerning free groups in these varieties. These latter theorems, in turn, are generalizations of classical theorems about absolutely free groups, i.e. free groups in the variety of all groups. We shall not be specifically concerned here, however, with absolutely parafree groups, although some of our results do hold in this case. In fact, we shall be dealing mainly with certain types of product varieties.

The bulk of the paper, following a brief preliminary section, consists of two chapters.

In Chapter I, we consider a well-known theorem of O. Schreier [10], asserting that a nontrivial normal subgroup of an absolutely free group of finite rank is finitely generated if and only if it is of finite index. G. Baumslag [3] considers possible generalizations of this theorem for certain relatively free groups and obtains the following result:

Let $\mathfrak{U}$ and $\mathfrak{B}$ be varieties of groups, $\mathfrak{U}$ not the variety of groups of order 1 , and let $F$ be a noncyclic freee group in $\mathfrak{U} \supseteqq$. Let $V(F)$ denote the (unique) minimal normal subgroup of $F$ such that $F / V(F)$ lies in $\Re$. Suppose that $N$ is a nontrivial normal subgroup of $F$ sucb that $F / V(F) N$ is infinite. Then $N$ is not finitely generated.

Note that in the case where $\mathfrak{U}$ and $\mathfrak{B}$ are both the varieties of all groups, Baumslag's theorem reduces to the "only if" part of Schreier's theorem. Our

Received by the editors August 17, 1970 and, in revised form, November 23, 1971 .

AMS 1970 subject classifications. Primary 20E10, 20E15, $20 \mathrm{E} 25$.

Key words and phrases. Parafree groups, varieties of groups, metabelian groups, lower central series, residually nilpotent groups, parabasis, left normed basic commutators.

(1) See the section on preliminaries that follows for references and definitions. 
contribution is to prove a form of Baumslag's theorem, replacing the free group $F$ by a parafree group $P$. However, we must make a drastic restriction on the varities $\mathfrak{U}$ and $\mathfrak{B}$. More precisely we shall prove:

Let $P$ be a parafree group of rank $\geq 2$ in the variety $\mathrm{Q}^{2}$ of all metabelian groups. Let $N$ be a normal subgroup of $P$ such that $\left|P / N \cdot \gamma_{2} P\right|$ is infinite. Then, either $N=1$ or $N$ is not finitely generated.

Notice that the free groups in $\mathrm{Q}^{2}$ are actually parafree, being residually nilpotent, and therefore our result can be viewed as a partial generalization of Baumslag's the orem.

In Chapter II, we shall prove the following theorem:

If $P$ is a parafree group in any variety $B$ containing the variety of all metabelian groups, and if $x$ and $y$ are any two elements of $P$ which are indepen. dent modulo $\gamma_{2} P$, then the commutator $[x, y]$ is not a proper power.

This result generalizes the analogous result for free groups, provided $B$ is either the variety of all groups (G. Baumslag [2]; W. Magnus, A. Karrass and D. Solitar [8]) or the variety of all metabelian groups (G. Baumslag, B. H. Neumann, H. Neumann and P. M. Neumann [6]). If $\mathfrak{U}$ is a variety which lies strictly between the variety of all groups and the variety of all metabelian groups, then a free group in $\mathfrak{U}$ is not necessarily a parafree group, and therefore our theorem does not apply directly to free groups in such varieties. Nevertheless, the result is still true for free groups $F$ in $\mathfrak{U}, \mathfrak{U} \geq \mathfrak{U}^{2}$. Indeed, we need only observe that $F / \gamma_{3} F$ is free in $\mathfrak{U}^{2}$.

We would like to mention that the techniques developed in this paper can be used to prove the following result:

A parafree group of infinite rank in the product variety $\mathrm{U}_{2} \mathrm{U}_{2}$ has a trivial center.

The proof is, unfortunately, very complicated, and, moreover, does not seem to generalize to other varieties (not even to $2 U_{p}, p$ an odd prime); thus it has not been included here. It should be noted, however, that the analogous result for free groups is known to be true for a class of varieties including all the $\mathrm{Ul}_{p}$, $p$ prime; see e.g., M. Auslander and R. C. Lyndon [1].

The content of this paper is based on the author's doctoral thesis, written under the direction of Professor G. Baumslag. I would like to take this opportunity to express my deep appreciation to Professor Baumslag for his guidance and encouragement.

Preliminaries. We refer the reader to H. Neumann [9] for the definition of varieties, product varieties, metabelian groups, nilpotent groups, residually nilpotent groups, lower central series of groups, relatively free groups, basic commutators and left normed basic commutators. (The definition of basic commutators in H. Neumann is given for finitely many generators only. When infinitely many 
generators are involved, only obvious modifications are required, see e.g. [11].)

Next we give certain definitions which were introduced by G. Baumslag and can be found in his papers [4] and [5].

Let $G$ be any group and let $\gamma_{n} G$ be the $n$th term of the lower central series. Then the sequence

$$
G / \gamma_{2} G, G / \gamma_{3} G, \cdots
$$

is called the lower central sequence of $G$. Let $H$ be another group. Then $H$ and $G$ have the same lower central sequence if there are isomorphisms $\theta_{k}$ from $G / \gamma_{k} G$ onto $H / \gamma_{k} H$ such that $\theta_{k}$ induces $\theta_{k-1}$ on $G / \gamma_{k-1} G$ to $H / \gamma_{k-1} H$ for $k=2,3, \ldots$.

A group $P$ is termed parafree in a variety $\mathcal{B}$ if

(i) $P \in \mathfrak{B}$,

(ii) $P$ is residually nilpotent, and

(iii) $P$ has the same lower central sequence as a free group in $\mathfrak{B}$.

A subset $X$ of a parafree group $P$ is called a parabasis for $P$ if $X$ freely generates $P$ modulo $\gamma_{2} P$. It follows that if $X$ is a parabasis of $P$, then $X$ freely generates $P$ modulo $\gamma_{n} P$ for $n \geq 2$.

We now list the notation which will be used throughout this paper.

Notation.

$x^{y}$ the transform $y^{-1} x y$ of $x$ by $y, x, y \in G$.

$x^{y_{1}+\ldots+y_{n}} x^{y_{1}} x^{y_{2}} \ldots x^{y_{n}}\left(x, y_{1}, \ldots, y_{n}\right.$ in the group $\left.G\right)$.

$\left[x_{1}, x_{2}\right]$ the commutator $x_{1}^{-1} x_{2}^{-1} x_{1} x_{2}$ of $x_{1}$ and $x_{2}$.

$\left[x_{1}, x_{2}, \ldots, x_{n}\right]$ the left normed commutator $\left[\left[x_{1}, x_{2}, \cdots, x_{n-1}\right], x_{n}\right]$.

$\left[x_{1}, k_{2} x_{2}, \cdots, k_{n} x_{n}\right]$ the left normed commutator with $k_{i}$ repetitions of $x_{i}$, $i=2, \cdots, n$, and $k_{i} \geq 0$. If $k_{i}=0$ the element $x_{i}$ does not appear at all.

$H \leq G H$ is a subgroup of $G$.

$\mathrm{gP}(X)$ the subgroup of $G$ generated by $X$, where $X$ is a subset of $G$.

$\mathrm{gP}_{G}(X)$ the normal subgroup $G$ generated by $X$.

$[A, B]$ the group generated by the commutators $[a, b], a \in A, b \in B$.

$\gamma_{n} G$ the $n$th term of the lower central series of a group $G$.

$|S|$ the cardinality of a set $S$.

$S \backslash T$ the set theoretical difference between $S$ and $T$, where $T$ is a subset of $S$. $\left\{s_{1}, \cdots, \hat{s}_{k}, \cdots, s_{n}\right\}$ the set $\left\{s_{1}, \cdots, s_{n}\right\} \backslash\left\{s_{k}\right\}$.

$\mathbf{Z}$ the set of integers.

$m \mid n \quad m$ divides $n(m, n \in \mathbf{Z})$.

$m \nmid n m$ does not divide $n(m, n \in \mathbf{Z})$.

$\mathscr{U}$ the variety of all abelian groups.

If $n$ variety of all abelian groups of exponent dividing $n(n \geq 2)$. 


\section{CHAPTER I}

1. The purpose of this chapter is to prove a generalization of a theorem of $G$. Baumslag [3]. Our result can be stated as follows:

Theorem 1. Let $P$ be a parafree froup of rank $\geq 2$ in the variety $\mathfrak{Q ⿱}^{2}$ of all metabelian groups, let $N$ be a normal subgroup of $P$ such that $\left|P / N \cdot \gamma_{2} P\right|$ is infinite. Then either $N=1$ or $N$ is not finitely generated.

The main tool used in the proof of the theorem is a formula expressing an arbitrary element of $P$ as an infinite product in an appropriate sense. (This formula will also be used in subsequent chapters.) Aside from several special cases, the proof of the theorem proceeds by contradiction, first reducing to the case when $P$ is parafree of rank 2. The assumption that $N$ is finitely generated, together with the other assumptions, leads to (several) systems of $(k+1)$ linear inhomogeneous real equations with $k$ unknowns (where $k+1=$ number of generators of $N)$. These systems must, on the one hand, always be solvable, but, on the other hand, we can arrange matters so that some of these systems are not solvable. This involves making certain estimates on the terms of the system.

The rest of Chapter $I$ is arranged as follows. In $\$ 2$ we prove our infinite product formula. Some technical lemmas concerning commutator formulas are derived in $\$ \$ 3$ and 4 , and in $\$ 5$ we introduce certain real functions which are used in making our estimates. Then, in $\$ 6$, we carry out our reduction to the rank 2 case, and, finally, we complete the proof in $\$ 7$.

2. Let $g$ be an arbitrary element of a group $G$. We write

$$
g=\prod_{i=1}^{\infty} g_{i} \text { iff }\left(\prod_{i=1}^{n} g_{i}\right)^{-1} g \in \gamma_{n} G, \text { for all } n .
$$

Now suppose that $G$ is residually nilpotent and let $g \in G$. Then we may choose elements $g_{1}, g_{2}, \cdots$ of $G$ such that $g_{i} \in \gamma_{i} G$ and such that

$$
g \equiv g_{1} \cdot g_{2} \cdot \cdots \cdot g_{n} \text { modulo } \gamma_{n+1} G .
$$

It follows immediately then that

$$
g=\prod_{i=1}^{\infty} g_{i}
$$

If one imposes certain extra conditions on the group $G$, then every element of $G$ can be written uniquely as a particular infinite product. This is the essence of the following discussion.

Let $G$ be a residually nilpotent group. For every $n \geq 1$, choose a collection $S_{n}$ of elements $g_{n, \alpha(n)} \in \gamma_{n} G, \alpha(n)$ ranging over some index set $\Lambda_{n}$, so that every element $g_{n} \in \gamma_{n} G$ can be uniquely expressed as a finite product of powers 
of the elements $g_{n, a(n)}$ modulo $\gamma_{n+1} G$. Thus, if we fix a well-ordering of $S_{n}$, we may write $g_{n}$ uniquely as

$$
g_{n}=\prod_{i=1}^{k} g_{n, a_{i}(n)}^{\rho_{n, a_{i}(n)}} \text { modulo } \gamma_{n+1} G,
$$

where $g_{n, a_{1}(n)}<\cdots<g_{n, a_{k}(n)}$ and $\rho_{n, a_{i}(n)} \in \mathbf{Z}$. Clearly, every element $g$ of $G$ can be written as follows:

$$
g=\prod_{n=1}^{\infty} \prod_{i=1}^{k(n)} g_{n, a_{i}(n)}^{\rho_{n, a_{i}(n)}}
$$

where $k(n)$ is a finite nonnegative integer, with the understanding that $\Pi_{i=1}^{0} g_{n, a_{i}(n)}=1$, and $g_{n, \alpha_{1}(n)}<g_{n, a_{2}(n)}<\cdots<g_{n, a_{k(n)}(n)}$. Suppose

$$
g=\prod_{n=1}^{\infty} \prod_{i=1}^{k(n)} g_{n, \alpha_{i}(n)}^{\rho_{n, \alpha_{i}(n)}}=\prod_{n=1}^{\infty} \prod_{j=1}^{l(n)} g_{n, \beta_{j}(n)}^{\rho_{n, \beta_{j}(n)}}
$$

where $0 \leq k(n)<\infty, 0 \leq l(n)<\infty, g_{n, a_{1}(n)}<\cdots<g_{n, a_{k(n)}(n)}$, and $g_{n, \beta_{1}(n)}<\cdots<$ $g_{n, \beta_{l(n)}(n)}$. Then for all $n \geq 1, k(n)=l(n)$ and $\alpha_{i}(n)=\beta_{i}(n)$ for all $n \geq 1$ and $1 \leq$ $i \leq k(n)$.

Let $P$ be a parafree group in the variety of all metabelian groups, with $x_{\lambda}$, $\lambda \in \Lambda$ as parabasis. For every $n \geq 1$, let $S_{n}$ be the set of all left normed basic commutators in $x_{\lambda}, \lambda \in \Lambda$ of weight $n$. (Of course, we assume that the sets $S_{n}$ are well-ordered.) Using a theorem of W. Magnus (see e.g. [9]) on the independence of the left normed basic commutators of weight $\geq 2$ in a free metabelian group, it follows immediately from the preceding discussion that every element of $P$ has a unique form as an infinite product of powers of left normed basic commutators in the $x_{\lambda}$ 's, arranged in an increasing order. This representation of elements of a parafree group in $\mathfrak{Q}^{2}$ will be used in Chapter II as well as in Chapter I.

3. Throughout this section we shall mainly deal with metabelian groups. The first four lemmas are known, and hence their proofs will be omitted.

Lemma 3.1. Let $G$ be any group, and let $x$ and $y$ be any two elements of $G$. Then

(i) $\left[x, y^{-1}\right]=\left([x, y]^{-1}\right)^{y^{-1}}$,

(ii) $\left[x^{-1}, y\right]=\left([x, y]^{-1}\right)^{x-1}$,

(iii) $\left[x^{-1}, y^{-1}\right]=[x, y]^{(x y)-1}$

Lemma 3.2 (P. Hall). Let $G$ be any group, and let $x, y$ and $z$ be arbitrary elements of $G$. Then

$$
\left[x, y^{-1}, z\right]^{y}\left[y, z^{-1}, x\right]^{z}\left[z, x^{-1}, y\right]^{x}=1 .
$$


Lemma 3.3. Let $G$ be any group, and let $x, y$, and $z$ be arbitrary elements of $G$. Then
(i) $[x y, z]=[x, z]^{y}[y, z]$,
(ii) $[x, y z]=[x, z][x, y]^{z}$,
(iii) $[x, y]^{z}=[x, y][x, y, z]$.

Moreover, if $G$ is a metabelian group, then

(iv) whenever $x \in \gamma_{2} G, x^{y z}=x^{z y}$,

(v) $\left[x^{r}, y^{s}\right]=[x, y]^{\left(1+x+\ldots+x^{r-1}\right)(1+y+\ldots+y s-1)}$

where $r$ and $s$ are positive integers.

Lemma 3.4. Let $G$ be any metabelian group, and let $x$ and $y$ be any two elements of G. Then $[y, k x, l y, m x]=[y,(k+m) x, l y]$ where $k \geq 1, l \geq 0$ and $m \geq 0$.

Proof. The proof can be found in H. Neumann [9, p. 96].

Lemma 3.5. Let $G$ be any residually nilpotent metabelian group. Let $g$ be an element of $\gamma_{2} G$, and let $b$ be any element of $G$. If we write $g=I_{i=2}^{\infty} g_{i}$ where $g_{i} \in \gamma_{i} G(i \geq 2)$, then
(i) $[g, b]=\Pi_{i=2}^{\infty}\left[g_{i}, b\right]$,
(ii) $g^{b}=\mathrm{II}_{i=2}^{\infty} g_{i}^{b}$.

Proof. Using Lemma 3.3 and the fact that $\gamma_{2} G$ is abelian, we get

$$
[g, b] \equiv \prod_{i=2}^{n}\left[g_{i}, b\right] \text { modulo } \gamma_{n+2} G \quad(n \geq 2) \text {. }
$$

Hence, $\left(\Pi_{i=2}^{n}\left[g_{i}, b\right]\right)^{-1}[g, b] \in \gamma_{n+2} G(n \geq 2)$. Thus, (i) is proved. The proof of (ii) is similar, and hence is omitted.

Before stating the next lemmas, we shall introduce certain notation.

Let $G$ be any group, and let $x$ be an arbitrary element of $G$. If $k$ and $l$ are any nonnegative integers, let $a(k, l, x)$ denote the following element of $\mathbf{Z}(G)$, the group ring of $G$ over the integers:

$$
\alpha(0, i, x)=x^{i} \text { for all } i \geq 0
$$

and inductively,

$$
\alpha(k, l, x)=\sum_{i=k-1}^{l-1} a(k-1, i, x) \text { for all } k>0 \text { and } l \geq 0,
$$

where our convention is that $\sum_{i=k-1}^{l-1} \alpha(k-1, i, x)=0$ if $l<k$. Thus, $\alpha(k, l, x)=0$ whenever $l<k$. Moreover, one should observe that $\alpha(k, k, x)=1$ for all $k \geq 0$.

Next, we shall denote by $m(s, t)$, where $s \geq 0, t \geq 0$, the following nonnegative integer: 


$$
m(0, t)=1 \text { for all } t \geq 0
$$

and inductively,

$$
m(s, t)=\sum_{i=s-1}^{t-1} m(s-1, i) \text { for all } s>0 \text { and } t \geq 0
$$

and again our convention is that whenever $t<s, \Sigma_{i=s-1}^{t-1} m(s-1, i)=0$. Thus $m(s, t)=0$ whenever $t<s$. Furthermore, it can easily be checked that $m(s, s)=1$ for all $s \geq 0$.

Now we are ready to proceed with the lemmas.

Lemma 3.6. Let $G$ be any metabelian group. If $x_{1}, x_{2}, \cdots, x_{n}$ and $z$ are arbitrary elements of $G$ such that $n \geq 2$, then

$$
\left[x_{1}, x_{2}, \cdots, x_{n}\right]^{\alpha(k, l, z)}=\left[x_{1}, x_{2}, \cdots, x_{n}\right]^{m(k, l)}\left[x_{1}, \cdots, x_{n}, z\right]^{\alpha(k+1, l, z)}
$$

where $k \geq 0$ and $l \geq 0$.

Proof. The proof is by induction on $k$. If $k=0$, we must show that

$$
\left[x_{1}, x_{2}, \cdots, x_{n}\right]^{\alpha(0, l, z)}
$$

By definition we get

$$
\alpha(0, l, z)=z^{l}
$$

and

$$
\alpha(1, l, z)=\sum_{i=0}^{l-1} \alpha(0, i, z) .
$$

We observe that if $l=0$ equation (1) holds trivially. Thus, assume that $l>0$. Using Lemma 3.3 and equations (2) and (3) we get

$$
\begin{aligned}
{\left[x_{1}, x_{2}, \cdots, x_{n}\right]^{z^{l}} } & =\left[x_{1}, x_{2}, \ldots, x_{n}\right]\left[x_{1}, x_{2}, \cdots, x_{n}, z^{l}\right] \\
& =\left[x_{1}, x_{2}, \cdots, x_{n}\right]\left[x_{1}, x_{2}, \cdots, x_{n}, z\right]^{1+z+\ldots+z l-1} \\
& =\left[x_{1}, x_{2}, \cdots, x_{n}\right]\left[x_{1}, x_{2}, \cdots, x_{r}, z\right]^{\alpha(1, l, z)} .
\end{aligned}
$$

Thus, equation (1) holds. Next, we shall assume that the result holds for some fixed $k>0$, and we shall prove that the following identity holds:

$$
\begin{aligned}
& {\left[x_{1}, x_{2}, \cdots, x_{n}\right]^{\alpha(k+1, l, z)} } \\
& \quad=\left[x_{1}, x_{2}, \cdots, x_{n}\right]^{m(k+1, l)}\left[x_{1}, x_{2}, \cdots, x_{n}, z\right]^{\alpha(k+2, l, z)} .
\end{aligned}
$$

Using the induction hypothesis and the fact that $\gamma_{2} G$ is abelian, we get 


$$
\begin{aligned}
{\left[x_{1}, x_{2}, \cdots, x_{n}\right]^{\alpha(k+1, l, z)}=\left[x_{1}, x_{2}, \cdots, x_{n}\right]^{\sum_{i=k}^{l-1} \alpha(k, i, z)} } & \\
& =\left[x_{1}, x_{2}, \cdots, x_{n}\right]^{\sum_{i=k}^{l-1} m(k, i)}\left[x_{1}, x_{2}, \cdots, x_{n}, z\right]^{\sum_{i=k}^{l-1} \alpha(k+1, i, z)}
\end{aligned}
$$

By definition we know that

$$
\sum_{i=k}^{l-1} m(k, i)=m(k+1, l)
$$

and

$$
\sum_{i=k}^{l-1} \alpha(k+1, i, z)=\sum_{i=k+1}^{l-1} \alpha(k+1, i, z)=\alpha(k+2, l, z) .
$$

Now, equation (4) follows immediately from equations (5), (6) and (7), and thus the proof of the lemma is complete.

Lemma 3.7. Let $G$ be any metabelian group. If $x_{1}, x_{2}, \ldots, x_{n}$ and $z$ are arbitrary elements of $G$ such that $n \geq 2$, then

where $l \geq 0$.

$$
\left[x_{1}, x_{2}, \ldots, x_{n}\right]^{z^{l}}=\prod_{i=0}^{l}\left[x_{1}, x_{2}, \ldots, x_{n}, i z\right]^{m(i, l)}
$$

Proof. The proof is by induction on $l$. Suppose $l=0$. Then

$$
\left[x_{1}, x_{2}, \cdots, x_{n}\right]^{z^{0}}=\left[x_{1}, x_{2}, \cdots, x_{n}\right]^{m(0,0)}
$$

because $m(0,0)=1$. Next, we shall assume that

$$
\left[x_{1}, x_{2}, \cdots, x_{n}\right]^{z^{j}}=\prod_{i=0}^{j}\left[x_{1}, x_{2}, \cdots, x_{n}, i z\right]^{m(i, j)} \text { for } j \leq l \text {, }
$$

where $l$ is a fixed positive integer, and we must show that

$$
\left[x_{1}, x_{2}, \cdots, x_{n}\right]^{z^{l+1}}=\prod_{i=0}^{l+1}\left[x_{1}, x_{2}, \cdots, x_{n}, i z\right]^{m(i, l+1)} .
$$

We first observe that by definition $z^{l}=\alpha(0, l, z)$. Thus, using Lemma 3.6 we get

$$
\begin{aligned}
{\left[x_{1}, x_{2}, \cdots\right.} & \left., x_{n}\right]^{z^{l+1}}=\left[x_{1}, x_{2}, \cdots, x_{n}\right]^{\alpha(0, l+1, z)} \\
& =\left[x_{1}, x_{2}, \cdots, x_{n}\right]^{m(0, l+1)}\left[x_{1}, x_{2}, \cdots, x_{n}, z\right]^{\alpha(1, l+1, z)} \\
& =\left[x_{1}, x_{2}, \cdots, x_{n}\right]^{m(0, l+1)}\left[x_{1}, x_{2}, \cdots, x_{n}, z\right]^{\sum_{j=0}^{l} \alpha(0, j, z)}
\end{aligned}
$$

Using the induction hypothesis, equation (3) and the fact that $\gamma_{2} G$ is abelian, it follows that 
(4)

$$
\begin{aligned}
{\left[x_{1}, x_{2}, \cdots, x_{n}\right]^{z^{l+1}=} } & {\left[x_{1}, x_{2}, \cdots, x_{n}\right]^{m(0, l+1)} } \\
& \cdot \prod_{j=0}^{l} \prod_{i=0}^{j}\left[x_{1}, x_{2}, \cdots, x_{n},(1+i) z\right]^{m(i, j)} \\
= & {\left[x_{1}, x_{2}, \cdots, x_{n}\right]^{m(0, l+1)} } \\
& \cdot \prod_{i=0}^{l}\left[x_{1}, x_{2}, \cdots, x_{n},(i+1) z\right]^{\sum_{j=1}^{l} m(i, j)}
\end{aligned}
$$

By the definition, we get

$$
m(i+1, l+1)=\sum_{j=1}^{l} m(i, j) .
$$

Hence it follows immediately from equations (4) and (5) that

$$
\begin{aligned}
{\left[x_{1}, x_{2}, \cdots, x_{n}\right]^{z^{l+1}}=} & {\left[x_{1}, x_{2}, \cdots, x_{n}\right]^{n(0, l+1)} } \\
& \cdot \prod_{i=0}^{l}\left[x_{1}, x_{2}, \cdots, x_{n},(1+i) z\right]^{m(i+1, l+1)} \\
= & \prod_{i=0}^{l+1}\left[x_{1}, x_{2}, \cdots, x_{n}, i z\right]^{m(i, l+1)}
\end{aligned}
$$

and the lemma is proved.

Lemma 3.8. Let $G$ be any metabelian group. If $x$ and $y$ are any elements of $G$, then

$$
[y, m x, n y]^{x l}=\prod_{i=0}^{l}[y,(m+i) x, n y]^{m(i, l)}
$$

where $m>0, n \geq 0$ and $l \geq 0$.

Proof. The proof of this lemma follows easily from Lemmas 3.4 and 3.7.

4. In this section, we shall deal with parafree groups of rank 2 in the variety of all metabelian groups. Using the ideas developed in $\$ 2$, it is easy to see that if $a$ and $b$ are a parabasis of $P$, then every element $p$ of $P$ can be written uniquely (modulo permutation of the commutators) as follows:

$$
p=a^{k_{0}, 0} b^{k_{0}, 1} \prod_{m \geq 1 ; n \geq 0}[b, m a, n b]^{k_{m, n}}
$$

here $k_{0,0}, k_{0,1}$ and $k_{m, n}$ (for $m \geq 1, n \geq 0$ ) are any integers. This representation of elements of $P$ will be used repeatedly in this section. 
The following lemma is essentially well known [7].

Lemma 4.1. Let $P$ be a parafree group of rank 2 in $\mathfrak{Q}^{2}$. If $a$ and $b$ form $a$ parabas is of $P$, then

$$
[b, m a, n b]^{b-1}=\prod_{i=0}^{\infty}[b, m a,(n+i) b]^{(-1)^{i}}
$$

where $m>0$ and $n \geq 0$.

Proof. We shall first show that for an arbitrary positive integer $k$ the following identity holds:

(1) $[b, m a, n b]^{b^{-1}}=\prod_{i=0}^{k-1}[b, m a,(n+i) b]^{(-1)^{i}} \cdot\left([b, m a,(n+k) b]^{(-1)^{k}}\right)^{b^{-1}}$.

The proof of equation (1) is by induction. Suppose $k=1$. Then we must show that

$$
[b, m a, n b]^{b^{-1}}=[b, m a, n b]\left([b, m a,(n+1) b]^{-1}\right)^{b^{-1}} .
$$

It follows from Lemmas 3.1 and 3.3 that

$$
\begin{aligned}
{[b, m a, n b]^{b^{-1}} } & =[b, m a, n b]\left[b, m a, n b, b^{-1}\right] \\
& =[b, m a, n b]\left([b, m a,(n+1) b]^{-1}\right)^{b^{-1}} .
\end{aligned}
$$

Next, suppose equation (1) holds for an arbitrary fixed $k>1$. Then we shall show that

$$
[b, m a, n b]^{b-1}=\prod_{i=0}^{k}[b, m a,(n+1) b]^{(-1)^{i}}
$$

$$
\cdot\left([b, m a,(n+k+1) b]^{(-1)^{k+1}}\right)^{b^{-1}} .
$$

Using the induction hypothesis, Lemmas 3.1 and 3.3 and the fact that $\gamma_{2} P$ is abelian we get

$$
\begin{aligned}
{[b, m a, n b]^{b-1}=\prod_{i=0}^{k-1}[b, m a,(n+i) b]^{(-1)^{i}} \cdot\left([b, m a,(n+k) b]^{(-1)^{k}}\right)^{b-1} } \\
\quad=\prod_{i=0}^{k}[b, m a,(n+i) b]^{(-1)^{i}}\left[b, m a,(n+k) b, b^{-1}\right]^{(-1)^{k}} \\
\quad=\prod_{i=0}^{k}[b, m a,(n+i) b]^{(-1)^{i}}\left\{\left([b, m a,(n+k+1) b]^{-1}\right)^{b^{-1}}\right\}^{(-1)^{k}} \\
=\prod_{i=0}^{k}[b, m a,(n+i) b]^{(-1)^{i}}\left([b, m a,(n+k+1) b]^{(-1)^{k+1}}\right)^{b^{-1}}
\end{aligned}
$$


Thus, equation (4) holds, and hence equation (1) is true for all $k \geq 0$. Since the commutator $\left([b, m a,(n+k) b]^{(-1)^{k}}\right)^{b-1}$ belongs to $\gamma_{m+n}+1+{ }_{k} P$, it follows immediately from the definition of an infinite product that

$$
[b, m a, n b]^{b^{-1}}=\prod_{i=0}^{\infty}[b, m a,(n+i) b]^{(-1)^{i}},
$$

and thus the lemma is proved.

Corollary. Let $P$ be a parafree group of rank 2 in $\mathfrak{Q}^{2}$. If $a$ and $b$ form $a$ parabasis of $P$, then

$$
\left[b, m a, n b, b^{-1}\right]=\prod_{i=1}^{\infty}[b, m a,(n+i) b]^{(-1)^{i}} .
$$

Proof. The proof follows immediately from Lemmas 3.3 and 4.1.

Lemma 4.2. Let $P$ be a parafree group of rank 2 in $\mathfrak{Q}^{2}$, with $a$ and $b$ as $a$ parabasis. Then

$$
\left[b, m a, n b, k b^{-1}\right]=\prod_{i=k}^{\infty}[b, m a,(n+i) b]^{\mu_{i}}
$$

where $m>0, n \geq 0, k \geq 1$ and the $\mu_{i}$ are integers depending on $k$.

Proof. The proof is by induction on $k$. If $k=1$, then the statement of Lemma 4.2 is reduced to the statement of the corollary of Lemma 4.1. Thus, suppose the lemma holds for an arbitrary fixed $k>1$. Then we shall show that

$$
\left[b, m a, n b,(k+1) b^{-1}\right]=\prod_{i=k+1}^{\infty}[b, m a,(n+i) b]^{\mu_{i}}
$$

for suitable $m>0, n \geq 0, k>1$ and $\mu_{i} \in \mathrm{Z}$. Using the induction hypothesis, Lemma 3.5 and the corollary of Lemma 4.1 , we get

$$
\begin{aligned}
{[b,} & \left.m a, n b,(k+1) b^{-1}\right]=\left[b, m a, n b, k b^{-1}, b^{-1}\right] \\
& =\left[\prod_{i=k}^{\infty}[b, m a,(n+i) b]^{\mu_{i}}, b^{-1}\right]=\prod_{i=k}^{\infty}\left[b, m a,(n+i) b, b^{-1}\right]^{\mu_{i}} \\
& \left.=\prod_{i=k}^{\infty}\left(\prod_{j=1}^{\infty}[b, m a,(n+i+j) b]^{(-1)^{j}}\right)\right)^{\mu_{i}}=\prod_{l=k+1}^{\infty}[b, m a,(n+l) b]^{\rho_{l}},
\end{aligned}
$$

where $\rho_{l}=\Sigma_{i+j=l ; j \geq 1, i \geq k}(-1)^{j} \mu_{i}$. Clearly, $\rho_{l} \in \mathbf{Z}$, and hence the lemma is proved.

Lemma 4.3. Let $P$ be a parafree group of rank 2 in $\mathfrak{Q}^{2}$, with $a$ and $b$ as a 
parabasis. Let $p$ be an arbitrary element of $\gamma_{2} P$. If we write

$$
p=\prod_{m>0 ; n \geq 0}[b, m a, n b]^{k_{m, n}}
$$

and

$$
p^{b^{s}}=\prod_{m>0 ; n \geq 0}[b, m a, n b]^{l_{m, n}}
$$

where $k_{m, n}, l_{m, n}$ and $s$ are integers, then $k_{m, 0}=l_{m, 0}$ for all $m$.

Proof. We shall first suppose that $s \geq 0$. Using Lemmas 3.5 and 3.7 and equation (1) we get

$$
\begin{aligned}
p^{b^{s}} & =\left(\prod_{m>0 ; n \geq 0}[b, m a, n b]^{k}, n\right)^{b^{s}}=\prod_{n>0 ; n \geq 0}\left([b, m a, n b]^{b^{s}}\right)^{k} m, n \\
& =\prod_{m>0 ; n \geq 0}\left(\prod_{i=0}^{s}[b, m a,(n+i) b]^{m(i, s)}\right)^{k_{m}, n} \\
& =\prod_{m>0 ; n \geq 0} \prod_{i=0}^{s}[b, m a,(n+i) b]^{k_{m, n} \cdot m(i, s)} .
\end{aligned}
$$

Now, we observe that the infinite product,

$$
\prod_{m>0 ; n \geq 0} \prod_{i=0}^{s}[b, m a,(n+i) b]^{k, m, n \cdot m(i, s)}
$$

contains a commutator ending with an $a$ if and only if $n=0$. Furthermore, if $n=0$, the commutator $[b, m a]$ appears to the power $k_{m, 0} \cdot m(0, s)=k_{m, 0}$, for all $m \geq 1$. Using the above fact, Lemma 2.1 and equation (2), it is clear that $k_{m, 0}=l_{m, 0}$ for all $m \geq 1$.

Next, suppose $s<0$, and put $t=-s$ and $c=b^{-1}$. Then, as in equation (3) we get

$$
\begin{aligned}
p^{b^{s}} & =p^{\left(b^{-1}\right)-s}=p^{c t} \\
& =\prod_{m>0 ; n \geq 0} \prod_{i=0}^{t}[b, m a, n b, i c]^{k_{m}, n \cdot m(i, t)} \\
& =\prod_{m>0 ; n \geq 0} \prod_{i=0}^{t}\left[b, m a, n b, i b^{-1}\right]^{k} m, n \cdot m(i, t) .
\end{aligned}
$$

Using Lemma 4.2 and equation (4) we get

$$
p^{b^{s}}=\prod_{m>0 ; n \geq 0} \prod_{i=0}^{t}\left(\prod_{j=i}^{\infty}[b, m a,(n+j) b]^{\mu_{j, i}}\right)^{k_{m, n} \cdot m(i, t)}
$$


where $\mu_{j, i}(j \geq 1)$ are integers such that $\mu_{0,0}=1$ and $\mu_{j, 0}=0$ whenever $j>0$. Thus, we observe that the infinite product on the right-hand side of equation (5) contains a commutator ending with an $a$ if and only if $n=0$. Furthermore, if $n=0$, then the commutator $[b, m a]$ appears to the power $k_{m, 0} \cdot m(0, t)=k_{m, 0}$, for all $m \geq 1$. Thus, as in the previous case, it follows that $k_{m, 0}=l_{m, 0}$ for all $m \geq 1$. Hence the lemma is proved.

Lemma 4.4. Let $P$ be a parafree group in $\mathcal{U}^{2}$ of rank 2, with $a$ and $b$ as $a$ parabasis. If

$$
p=b^{s} \prod_{m>0 ; n \geq 0}[b, m a, n b]^{k_{m, n}},
$$

then $p^{a t}=b^{s}[b, a]^{s t+k_{1}, 0}$. П commutators of higher weight, where $s \geq 0$, and $t \geq 0$.

Proof. We first note that $p \equiv b^{s}[b, a]^{k} 1,0$ modulo $\gamma_{3} P$. Thus, using Lemma 3.3 we get

$$
\begin{aligned}
p^{a^{t}} & \equiv b^{s}\left[b^{s}, a^{t}\right]\left([b, a]^{k_{1}, 0}\right)^{a t} \equiv b^{s}[b, a]^{s t}[b, a]^{k_{1}, 0} \\
& \equiv b^{s}[b, a]^{s t+k_{1}, 0} \text { modulo } \gamma_{3} P .
\end{aligned}
$$

Hence the lemma is proved.

5. Lemma 5.1. If we put

$$
f_{n}(x)=\{x-(n-1)\}^{n} / n !
$$

where $x \in \mathbf{R}$ and $n$ is an integer $\geq 0$, then $m(n, t) \geq f_{n}(t)$ for all $t \geq n$.

Proof. The proof is by induction on $n$. Suppose $n=0$. Then we must show that

$$
m(0, t) \geq f_{0}(t) \text { for all } t \geq 0
$$

But, by the definitions, we get

and

$$
m(0, t)=1 \quad \text { for all } t \geq 0
$$

Now, equation (1) follows immediately from equations (2) and (3). Next, we shall assume that the lemma is true for an arbitrary fixed $n$, and we shall show that

$$
m(n+1, t) \geq f_{n+1}(t) \text { for all } t \geq n+1 \text {. }
$$

We first observe that $f_{n}(x)$ is an increasing function for all $x \geq(n-1)$, and thus the sum, $\Sigma_{i=n}^{t-1} f_{n}(i)(t>n)$, is an upper approximation of the integral $\int_{n-1}^{t-1} f_{n}(x) d x$. But 


$$
\begin{aligned}
\int_{n-1}^{t-1} f_{n}(x) d x & =\int_{n-1}^{t-1} \frac{\{x-(n-1)\}^{n}}{n !} d x \\
& =\left.\frac{\{x-(n-1)\}^{n+1}}{(n+1) !}\right|_{-n-1} ^{t-1}=\frac{(t-n)^{n+1}}{(n+1) !}
\end{aligned}
$$

Using the induction hypothesis we know that

$$
f_{n}(i) \leq m(n, i) \text { for } i \geq n \text {. }
$$

Thus, using the upper approximation together with equations (5) and (6), we get

$$
\frac{(t-n)^{n+1}}{(n+1) !} \leq \sum_{i=n}^{t-1} f_{n}(i) \leq \sum_{i=n}^{t-1} m(n, i)=m(n+1, t)
$$

for all $t \geq n+1$. But, by definition,

$$
f_{n+1}(t)=(t-n)^{n+1} /(n+1) !
$$

Now, the lemma follows immediately from equations (7) and (8).

Lemma 5.2. If we put $g_{0}(x)=1$ and $g_{n}(x)=x^{n} / n !$, where $x \in \mathbf{R}$ and $n$ is an integer $>0$, then $g_{n}(t) \geq m(n, t)$ for all $t \geq n$.

Proof. The proof of Lemma 5.2 is similar to the proof of Lemma 5.1 and hence is omitted.

6. Lemma 6.1. Let $P$ be a parafree group in the variety $\mathfrak{U}^{2}$. If $N$ is a normal subgroup of $P$ such that $N<\gamma_{2} P$ and $\left|P / N \gamma_{2} P\right|$ is infinite, then either $N=1$ or $N$ is not finitely generated.

Proof. Suppose $N \neq 1$ and $N$ is finitely generated. Since $\gamma_{2} P$ is a torsion free abelian group, $N$ is free abelian of finite rank. Now, let $a$ be any element of $N$ different from the identity. As the center of $P$ is 1 , it follows that there is an element $b$ of $P$ such that $[a, b] \neq 1$. Using a theorem of G. Baumslag [3] it follows that

$$
H=\operatorname{gP}\left(a^{b^{i}} \mid i=0,1,2, \ldots\right)
$$

is free abelian of infinite rank. But $H \leq N$, and hence we arrive at a contradiction. Thus, the lemma is proved.

Lemma 6.2. Suppose Theorem I is true whenever $P$ is a parafree group of rank 2. Then Theorem $I$ is true for an arbitrary, noncyclic, parafree group $P$.

Proof. We shall first assume that $\mathrm{P} / \mathrm{N} \gamma_{2} P$ is periodic. Then, since $\left|P / N \gamma_{2} P\right|$ is finite, it follows that $P$ is of infinite rank. Let $\eta$ be the natural homomorphism of $P$ onto $P / \gamma_{2} P$. Then, clearly, it is enough to show that $N \eta$ 
is not finitely generated. Next, suppose $N \eta$ is finitely generated. Then, $P \eta / N \eta$ is not periodic, because $P \eta$ is a free abelian group of infinite rank. Thus we have arrived at a contradiction.

Next, we shall assume that $P / N \gamma_{2} P$ is not periodic. Using Lemma 6.1 , it is enough to consider the case when $N$ is not a subgroup of $\gamma_{2} P$. Now, suppose $N$ is finitely generated. Then using the basis theorem for free abelian groups, there exists a parabasis, $X$, of $P$ and a finite subset, $\left\{x_{1}, \cdots, x_{n}\right\}$, of $X$ such that the group $N \gamma_{2} P$ is generated by the elements $x_{1}^{\alpha_{1}}, x_{2} \alpha_{2}, \ldots, x_{n}^{\alpha}$ modulo $\gamma_{2} P$, where $\alpha_{i} \in \mathbf{Z}(i=1,2, \cdots, n)$. Furthermore, since $P / N \gamma_{2} P$ is not periodic and $N$ is not a subgroup of $\gamma_{2} P$, it is easy to see that $X$ and its subset $\left\{x_{1}, \cdots, x_{n}\right\}$ can be chosen so that $\alpha_{1}=0, \alpha_{2} \neq 0$ and $x_{2}^{a_{2}} \notin y_{2} P$. Now, put

$$
K=g \mathrm{P}_{P}\left(X \backslash\left\{x_{1}, x_{2}\right\}\right)
$$

and

$$
J=\bigcap_{n} \gamma_{n} P \cdot K .
$$

Using a theorem of G. Baumslag [3], we know that $P / J$ is a parafree group of rank 2. Let $\mu$ be the natural homomorphism of $P$ onto $P / J$. Then, it is left to show that

$$
N \mu \neq 1
$$

and

$$
\left|P \mu / N \mu \gamma_{2}(P \mu)\right| \text { is infinite. }
$$

We shall first show that equation (3) holds. We know that there exists an element $n$ of $N$ such that $n=x_{2}^{a}{ }^{2} \cdot p^{\prime}$ where $p^{\prime} \in \gamma_{2} P$. Now, $P / K / \gamma_{2}(P / K)$ is free abelian of rank 2 , freely generated by $x_{1} K$ and $x_{2} K$ modulo $\gamma_{2}(P / K)$. So let $\rho$ be the homomorphism of $P / K / \gamma_{2}(P / K)$ into $P / \gamma_{2} P$ defined by

$$
x_{i} K \cdot\left(\gamma_{2}(P / K)\right) \rho=x_{i} \gamma_{2} P \quad(i=1,2) .
$$

Then, clearly, $n \cdot K\left(\gamma_{2}(P / K)\right) \rho \neq 1$ and thus $n \notin \gamma_{2} P \cdot K \supset J$. Hence $N \mu \neq 1$.

Next, we shall prove (4). Let $\nu$ be the homomorphism of $P / J / \gamma_{2}(P / J)$ into $P / \gamma_{2} P$ defined by

Now clearly

$$
x_{i} J\left(\gamma_{2} P / J\right) \nu=x_{i} y_{2} P \quad(i=1,2) .
$$

$$
\left\{(N \mu) \cdot\left(\gamma_{2}(P / J)\right)\right\}_{\nu}=\left\{\mathrm{gP}\left(x_{2}^{\alpha_{2}} J\right) \cdot\left(\gamma_{2}(P / J)\right)\right\}_{\nu}=\mathrm{gP}\left(x_{2}^{\alpha_{2}} \cdot \gamma_{2} P\right) .
$$

Suppose $x_{1}^{k} \cdot J \in(N \mu) \cdot\left(\gamma_{2}(P \mu)\right)$ for some $k \neq 0$. Then using equation (5) we get $x_{1}^{k} \gamma_{2} P \in \mathrm{gP}\left(x_{2}^{\alpha} \gamma_{2} P\right)$. But $x_{1}$ and $x_{2}$ are independent modulo $\gamma_{2} P$. Hence $x_{1}^{k} \mu \notin(N \mu) \cdot\left(\gamma_{2}(P \mu)\right)$ for all $k \neq 0$, and thus (4) is true. 
4.7. Lemma 7.1. Let $P$ be a positive group of rank 2 in the variety $\mathfrak{U}^{2}$. If $N$ is a nontrivial normal subgroup of $P$ such that $\left|P / N \cdot \gamma_{2} P\right|$ is infinite, then $N$ is not finitely generated.

Proof. It follows from Lemma 6.1 that we can assume that $N$ is not a subgroup of $\gamma_{2} P$. Thus $N \gamma_{2} P / \gamma_{2} P \neq 1$. Using the basis theorem for free abelian groups, it follows that we can choose a parabasis, $\{a, b\}$, of $P$ such that $a^{r}$ and $b^{s}$, where $s$ and $r$ are integers, generates $N \cdot \gamma_{2} P$ modulo $\gamma_{2} P$. Since $N \gamma_{2} P / \gamma_{2} P \neq$ 1 and $\left|P / N \gamma_{2} P\right|$ is infinite, without loss of generality we shall assume that $s \neq 0$ and $r=0$. Furthermore, we shall assume that $s>0$. Suppose $N$ is finitely generated, i.e. suppose

$$
N=\mathrm{gP}\left(b_{1}, \cdots, b_{k}\right) .
$$

Clearly $b_{i}=b^{s n_{i}} b_{i}^{\prime}$, where $n_{i} \in \mathbf{Z}$ and $b_{i}^{\prime} \in \gamma_{2} P$ for all $1 \leq i \leq k$. Since $b^{s}$. $\gamma_{2} P$ is an element of $N \gamma_{2} P / \gamma_{2} P$, there is an element $p$ of $N$ such that $p=b^{s} p^{\prime}$, where $p^{\prime} \in \gamma_{2} P$. Put

$$
g_{i}=(p)^{-n_{i}} b_{i} \text { for } 1 \leq i \leq k .
$$

Clearly, $g_{i} \in \gamma_{2} P$ for $1 \leq i \leq k$. Using equations (1) and (2) we get

(3) $\quad N=\mathrm{gP}\left(b_{1}, \cdots, b_{k}\right)=\mathrm{gP}\left(p, g_{1}, \cdots, g_{k}\right)=\mathrm{gP}(p) \cdot \mathrm{gP}\left(g_{1}^{b^{s t}}, \cdots, g_{k}^{b^{s t}} \mid t \in \mathrm{Z}\right)$.

Now, the proof of the theorem is broken into two cases, depending on whether $k=$ 0 or $k \neq 0$.

Case 1. Suppose $k=0$. Then $N=\mathrm{gP}\left(b^{s} p^{\prime}\right)$. Since $N$ is a normal subgroup of $P$, the element $p^{a}$ belongs to $N$. Thus we must have

$$
p^{a}=p^{l} \text { where } l \in \mathbf{Z} .
$$

We also know that $p=b^{s} p^{\prime}$ can be written as follows:

$$
p=b^{s} \prod_{m>0 ; n>0}[b, m a, n b]^{k_{m}, n},
$$

where $k_{m, n} \in \mathbf{Z}$. Using equation (5) and Lemma 4.4, we get

$$
p^{a} \equiv b^{s}[b, a]^{s+k_{1}, 0} \text { modulo } \gamma_{3} P \text {. }
$$

Moreover, $p^{l} \equiv b^{l s}$ modulo $\gamma_{2} P$. Hence $l=1$. Thus, by equation (4) $p^{a} \equiv p$ modulo $\gamma_{3} P$. But this implies that $s=0$, and thus leads to a contradiction.

Case 2. Suppose $k \neq n$. Then

$$
N=\mathrm{gP}(p) \cdot \mathrm{gP}\left(g_{1}^{b^{s t}}, \cdots, g_{k}^{b^{s t}} \mid t \in \mathbf{Z}\right)
$$

where $g_{i} \in \gamma_{2} P, 1 \leq i \leq k$. Put

$$
g_{i}=\prod_{m>0 ; n \geq 0}[b, m a, n b]^{l_{m, n}^{(i)}} \quad \text { for } \quad 1 \leq i \leq k,
$$


where $l_{m, n}^{(i)} \in \mathbf{Z}$, and

$$
g_{i}^{b^{s t}}=\prod_{m>0 ; n \geq 0}[b, m a, n b]^{l_{m, n}^{(i, t)}}
$$

where $l_{m, n}^{(i, t)} \in \mathbf{Z}$. It follows immediately from Lemma 4.3 and equations (8) and (9) that $l_{m, 0}^{(i)^{m, n}}=l_{m, 0}^{(i, t)}$ for all $m>0,1 \leq i \leq k$ and $t \in \mathbf{Z}$. Next, we shall show that for at least one $i, 1 \leq i \leq k, l_{1,0}^{(i)} \neq 0$. Since $p^{a} \in N$ we can write

$$
p^{a}=p^{l} \prod_{i=1}^{k} \prod_{j=1}^{r} g_{i}^{b^{s \cdot t_{i, j}}}
$$

where $t_{i, j}, r_{i}$ and $l$ are integers. Using equation (6) and (10), it follows that $l=1$. Moreover, using equations (9) and (10), we get

$$
p^{a}=b^{s}[b, a]^{k} 1,0+\sum_{i=1}^{k} r_{i} l_{l, 0}^{(i)} \cdot \prod \text { commutators of higher weight. }
$$

On the other hand, using Lemma 4.4, we get

$$
p^{a}=b^{s}[b, a]^{s+k_{1}, 0} \cdot \prod \text { commutators of higher weight. }
$$

Hence, the following identity holds

$$
s=\sum_{i=1}^{k} r_{i} l_{1,0}^{(i)}
$$

But $s \neq 0$, and so there is an $i, 1 \leq i \leq k$ such that $l_{1,0}^{(i)} \neq 0$. Without loss of generality we may assume that $l_{1,0}^{(1)} \neq 0$. Next, we shall write the element $g_{1}^{a u}$ $(u>0)$ in two ways. We shall then derive, for each $u$, a system of linear inhomogeneous equations by comparing the two different representations of $g_{1}^{a}$. On the one hand, using Lemma 3.5, we get

$$
g_{1}^{a^{u}}=\left(\prod_{m>0 ; n \geq 0}[b, m a, n b]^{l_{m, n}^{(1)}}\right)^{a^{u}}=\prod_{m>0 ; n \geq 0}\left([b, m a, n b]^{\left.a^{u}\right)^{l_{m, n}^{(1)}} .}\right.
$$

Using equation (14) and Lemma 3.8, we get, for every $u>0$,

$$
\begin{aligned}
g_{1}^{a^{u}}= & \prod_{m>0 ; n \geq 0}\left(\prod_{i=0}^{u}[b,(m+i) a, n b]^{m(i, u)}\right)^{l_{m, n}^{(1)}} \\
= & \prod_{j=1}^{k+1}[b, j a]^{\sum_{i=1}^{j} l_{i, 0}^{(1)} m(j-i, u)} \\
& \prod_{m>0 ; n \geq 0}\left(\prod_{i=0}^{u}[b,(m+i) a, n b]^{m(i, u)}\right)^{l_{m, n}^{(1)}}, \\
& (m+i, n) \in\{(1,0), \ldots(k+1,0)\} .
\end{aligned}
$$

On the other hand, since $N$ is normal, we get 


$$
g_{1}^{a^{u}}=p^{l_{u}} \prod_{i=1}^{k} \prod_{j=1}^{r_{i, u}} g_{i}^{b^{s t_{i, j}}}
$$

where $u>0, l_{u} \in \mathbf{Z}, r_{i, u} \in \mathbf{Z}$ and $t_{i, j} \in \mathbf{Z}$. Since $g_{1} \in \gamma_{2} P$, it follows that $l_{u}=$ 0 for all $u>0$. Thus, using equations (9) and (16) and Lemma 4.3, we get

$$
g_{1}^{a^{u}}=\prod_{i=1}^{k} \prod_{j=1}^{r_{i, u}} \prod_{m>0 ; n \geq 0}[b, m a, n b]^{l_{m, n}^{\left(i, t_{i, j}\right)}}
$$

$$
\begin{aligned}
=\prod_{j=1}^{k+1}\left([b, j a]^{\left.\sum_{i=1}^{k} l_{j, 0}^{(i)} r_{i, u}\right)} \prod_{i=1}^{k} \prod_{j=1}^{r_{i, u}} \prod_{m>0 ; n \geq 0}[b, m a, n b]^{\left.l_{m, n}^{(i, t i, j}\right)},\right. \\
\quad(m, n) \notin\{(1,0), \ldots,(k+1,0)\} .
\end{aligned}
$$

Using equations (15) and (17) and Lemma 2.1, we get for each $u>0$ the following $k+1$ equations:

$$
\sum_{i=1}^{k} l_{j, 0}^{i} r_{i, u}=\sum_{i=1}^{j} l_{1,0}^{1} m(j-i, u) \quad \text { for } j=1,2, \ldots, k+1 .
$$

Now, consider a real vector space of dimension $k$, generated by $i, u, i=1,2, \ldots$, $k$. Then the $k+1$ vectors $\sum_{i=0}^{k} l_{j, 0}^{(i)} r_{i, u}(j=1, \cdots, k+1)$ must be linearly dependent. Hence, there exist nonzero real numbers $n_{1}, \cdots, n_{k+1}$ such that

$$
\sum_{j=1}^{k+1} n_{j} \sum_{i=1}^{k} l_{j, 0}^{(i)} r_{i, u}=0
$$

Using equations (18) and (19), it follows that

$$
\sum_{j=1}^{k+1} n_{j} \sum_{i=1}^{j} l_{i, 0}^{(1)} m(j-i, u)=0 \text {. }
$$

Next, we shall show that if $u$ is sufficiently large, equation (20) does not hold.

It follows from Lemma 5.1 and 5.2 that whenever $u>0$,

$$
u^{j-i} /(j-i) ! \geq m(j-i, u) \geq\{u-(j-i-1)\}^{j-i} /(j-i) ! \text {. }
$$

We first note that for every $u>0$ we get

$$
\begin{aligned}
& \left|n_{k+1} \sum_{i=2}^{k+1} l_{i, 0}^{(1)} m(k+1-i, u)+\sum_{j=1}^{k} n_{j} \sum_{i=1}^{j} l_{i, 0}^{(1)} m(j-i, u)\right| \\
& \leq\left|n_{k+1}\right| \sum_{i=2}^{k+1}\left|l_{i, 0}^{(1)}\right| m(k+1-i, u)+\sum_{j=1}^{k}\left|n_{j}\right| \sum_{i=1}^{j}\left|l_{i, 0}^{(1)}\right| m(j-i, u) \\
& \quad \leq\left|n_{k+1}\right| \sum_{i=2}^{k+1}\left|l_{i, 0}^{(1)}\right| \frac{u^{k+1-i}}{(k+1-i) !}+\sum_{j=1}^{k}\left|n_{j}\right| \sum_{i=1}^{j}\left|l_{i, 0}^{(1)}\right| \frac{u^{j-i}}{(k+1-i) !} \\
& \quad \leq\left|n_{k+1}\right| \sum_{i=2}^{k+1}\left|l_{i, 0}^{(1)}\right| u^{k+1-i}+\sum_{j=1}^{k}\left|n_{j}\right| \sum_{i=1}^{j}\left|l_{i, 0}^{(1)}\right| u^{j-i} .
\end{aligned}
$$


Using equation (22), it follows immediately that there exist positive integers $K_{1}$ and $N_{1}$ such that whenever $u>N_{1}$ we get

$$
\left|n_{k+1} \sum_{i=2}^{k+1} l_{i, 0}^{(1)} m(k+1-i, u)+\sum_{j=1}^{k} n_{j} \sum_{i=1}^{j} l_{i, 0}^{(1)} m(j-i, u)\right| \leq K_{1} u^{k-1} \text {. }
$$

Moreover, we note that

$$
\left|n_{k+1} l_{1,0}^{(1)} m(k, u)\right| \geq\left|n_{k+1} l_{1,0}^{(1)}\right| \frac{(u-k)^{k}}{k !} .
$$

Put $\left|n_{k+1} l_{1,0}^{(1)}\right|=K_{2}$. Then $K_{2} \neq 0$ because $n_{k+1} \neq 0$ and $l_{1,0}^{(1)} \neq 0$. Next, we observe that there exists a positive integer, $N_{2}$, such that $N_{2}>N_{1}$ and such that whenever $u>N_{2}$ we get

$$
K_{2}\left((u-k)^{k} / k !\right)>K_{1} u^{k-1} \text {. }
$$

Thus it follows from equations (23), (24) and (25) that whenever $u>N_{2}$ we get

$$
\begin{aligned}
& \left|\sum_{j=1}^{k+1} n_{j} \sum_{i=1}^{j} l_{i, 0}^{(1)} m(j-i, u)\right| \\
& \geq\left|n_{k+1} l_{1,0}^{(1)} m(k, u)\right| \\
& \quad-\left|n_{k+1} \sum_{i=2}^{k+1} l_{1,0}^{(1)} m(k+1-i, u)+\sum_{j=1}^{k} n_{j} \sum_{i=1}^{j} l_{i, 0}^{(1)} m(j-i, u)\right| \\
& \quad \geq K_{2} \frac{(u-k)^{k}}{k !}-K_{1} u^{k-1}>0 .
\end{aligned}
$$

Hence, equation (20) does not hold for every $u>N_{2}$. Thus Lemma 6.1, and hence Theorem I, is proved.

\section{CHAPTER II}

1. In this chapter, we shall generalize a theorem of G. Baumslag, B. H. Neumann, H. Neumann and P. M. Neumann [6] concerning free groups in the variety of all metabelian groups to parafree groups in any variety larger than the variety of all metabelian groups. Precisely, we shall prove

Theorem II. Let $P$ be a parafree group in any variety $B$ containing the variety $\mathfrak{U}^{2}$ of all metabelian groups. If $x$ and $y$ are any two elements of $P$ which are independent modulo $\gamma_{2} P$, then there is no element $z \in P$ such that $[x, y]=$ $z^{m}, m>1$.

Proof. Let us assume, to begin with, that the theorem is true whenever $P$ is a parafree group of rank 2 in $\mathfrak{U}^{2}$. We shall return to this important special case later. 
Let now $P$ be an arbitrary noncyclic parafree group $P$ in $2^{2}$ and suppose there exist elements $x, y$ and $z$ of $P$ such that $x$ and $y$ are independent modulo $\gamma_{2} P$ and $[x, y]=z^{m}$ for some $m>1$. Now, since $x$ and $y$ are independent modulo $\gamma_{2} P$, there exists a parabasis $X$ of $P$ which includes two elements $a$ and $b$ such that $x$ and $y$ lie in $\mathrm{gP}(a, b)$ modulo $\gamma_{2} P$. Next, let

$$
K=g P_{P}(X \backslash\{a, b\})
$$

and

$$
J=\bigcap_{n}\left(\gamma_{n} P \cdot K\right)
$$

Using a theorem of G. Baumslag, we know that $P / J$ is a parafree group of rank 2. Now, if $\eta$ denotes the natural homomorphis $m$ of $P$ onto $P / J$, then, clearly $x \eta$ and $y \eta$ are independent modulo $\gamma_{2}(P / J)$ and $[x \eta, y \eta]=(z \eta)^{m}$, where $m>1$, thus leading to a contradiction.

Next, let $\mathcal{B}$ be any variety containing $\mathfrak{U}^{2}$ and let $P$ be any noncyclic parafree group in $\mathfrak{B}$. As before, suppose there exist elements $x, y$ and $z$ of $P$ such that $x$ and $y$ are independent modulo $\gamma_{2} P$ and $[x, y]=z^{m}$ for some $m>1$. Now let

$$
J=\bigcap_{n}\left(\gamma_{n} P \cdot \gamma_{3} P\right)
$$

Then it is easy to see that $P / J$ is a noncyclic parafree group in $\mathfrak{Q}^{2}$, i.e. is of the type just considered. But notice that if $\eta$ denotes the natural homomorphism of $P$ onto $P / J$, then $x \eta$ and $y \eta$ are independent modulo $\gamma_{2}(P / J)$ and $[x \eta, y \eta]=$ $(z \eta)^{m}, m>1$, and thus we have again a contradiction.

It remains to consider the case where $P$ is a parafree group of rank 2 in $\mathscr{U}^{2}$. We shall require a number of technical lemmas.

Le mma 2.1. Let $G$ be any metabelian group. If $x$ and $y$ are arbitrary elements of $G$, then

$$
[y, x]^{y^{s} x^{t}}=\prod_{j=0}^{s}\left(\prod_{i=0}^{t}[y,(1+i) x, j y]^{m(i, t)}\right)^{m(j, s)}
$$

where $s \geq 0$ and $t \geq 0$.

Proof. Using Lemma 3.3 of Chapter I we get

$$
[y, x]^{y^{s} x^{t}}=[y, x]\left[y, x, y^{s} x^{t}\right]=[y, x]\left[y, x, x^{t}\right]\left[y, x, y^{s}\right]^{x^{t}} .
$$

Using equation (1) and Lemmas 3.6 and 3.7 of Chapter I we get 
(2)

$$
\begin{aligned}
{[y, x]^{y^{s} x^{t}} } & =[y, x] \prod_{i=1}^{t}[y,(1+i) x]^{m(i, t)}\left(\prod_{j=1}^{s}[y, x, j y]^{m(j, s)}\right)^{x^{t}} \\
& =\prod_{i=0}^{t}[y,(1+i) x]^{m(i, t)} \prod_{j=1}^{s}\left([y, x, j y]^{x^{t}}\right)^{m(j, s)} \\
& =\prod_{i=0}^{t}[y,(1+i) x]^{m(i, t)} \prod_{j=1}^{s}\left(\prod_{i=0}^{t}[y,(1+i) x, j y]^{m(i, t)}\right)^{m(j, s)} \\
& =\prod_{j=0}^{s}\left(\prod_{i=0}^{t}[y,(1+i) x, j y]^{m(i, t)}\right)^{m(j, s)}
\end{aligned}
$$

Thus, the lemma is proved.

Lemma 2.2. Let $G$ be any metabelian group. If $x$ and $y$ are arbitrary elements of $G$, then

$$
\left[y^{k}, x^{l}\right]=\prod_{0 \leq s \leq k-1 ; 0 \leq t \leq l-1} \prod_{j=0}^{s}\left(\prod_{i=0}^{t}[y,(1+i) x, j y]^{m(i, t)}\right)^{m(j, s)}
$$

where $k \geq 0$ and $l \geq 0$.

Proof. Using Lemma 2.3 of Chapuer I and Lemma 2.1 of Chapter II we get

$$
\begin{aligned}
{\left[y^{k}, x^{l}\right] } & =[y, x]^{\left(1+y+\ldots+y^{k-1}\right)(1+x+\ldots+x l-1)}=\prod_{0 \leq s \leq k-1 ; 0 \leq t \leq l-1}[y, x]^{y^{s} x^{t}} \\
& =\prod_{0 \leq s \leq k-1 ; 0 \leq t \leq l-1} \prod_{j=0}^{s}\left(\prod_{i=0}^{t}[y,(1+i) x, j y]^{m(i, t)}\right)^{m(j, s)} .
\end{aligned}
$$

Hence, the lemma is proved.

Lemma 2.3. Let $G$ be any metabelian group. If $x$ and $y$ are elements of $G$ sucb that

$$
x=g_{1}^{l} \prod_{i=2}^{\infty} g_{i}
$$

and

(2)

$$
y=b_{1}^{k} \prod_{i=2}^{\infty} b_{i}
$$

where $g_{i} \in \gamma_{i} G$ and $b_{i} \in \gamma_{i} G, i \geq 1$, and $l, k>0$, then

$$
[x, y]=\left[g_{1}^{l}, b_{1}^{k}\right] \prod_{i=2}^{\infty} b_{i}^{-\varepsilon_{1}^{l}} \prod_{i=2}^{\infty} z_{i}^{b_{1}^{k}} \prod_{i=2}^{\infty} b_{i} \prod_{i=2}^{\infty} g_{i}^{-1} \text {. }
$$


Proof. Put

$$
g^{\prime}=\prod_{i=2}^{\infty} g_{i}
$$

and

$$
b^{\prime}=\prod_{i=2}^{\infty} b_{i} .
$$

Clearly $g^{\prime}$ and $b^{\prime}$ belong to $\gamma_{2} G$. Now, using Lemma 3.3 of Chapter I and the fact that $\gamma_{2} G$ is abelian we get

$$
\begin{aligned}
{[x, y] } & =\left[g_{1}^{l} g^{\prime}, b_{1}^{k} b^{\prime}\right]=\left[g_{1}^{l}, b_{1}^{k}\right]\left[g_{1}^{l}, b^{\prime}\right]\left[g^{\prime}, b_{1}^{k}\right] \\
& =\left[g_{1}^{l}, b_{1}^{k}\right]\left(b^{\prime-1}\right)^{g_{1}^{l}} b^{\prime} g^{\prime-1} g^{b_{1}^{k}} .
\end{aligned}
$$

Using Lemma 3.5 of Chapter I we know that

$$
g^{\prime-1}=\prod_{i=2}^{\infty} g_{i}^{-1}
$$

and

$$
b^{\prime-1}=\prod_{i=2}^{\infty} b_{i}^{-1}
$$

Thus, using equations (3), (4) and (5) and Lemma 3.5 of Chapter I we get

$$
[x, y]=\left[g_{1}^{l}, b_{1}^{k}\right] \prod_{i=2}^{\infty} b_{i}^{-g_{1}^{l}} \prod_{i=2}^{\infty} b_{i} \prod_{i=2}^{\infty} g_{i}^{-1} \prod_{i=2}^{\infty} g_{i}^{b_{1}^{k}}
$$

$$
=\left[g_{1}^{l}, b_{1}^{k}\right] \prod_{i=2}^{\infty} b_{i}^{-g_{1}^{l}} \prod_{i=2}^{\infty} g_{i}^{b_{1}^{k}} \prod_{i=2}^{\infty} b_{i} \prod_{i=2}^{\infty} g_{i}^{-1} .
$$

Thus, the lemma is proved.

Now we are ready to proceed with the proof of Theorem II.

Suppose then that there exist elements $x, y$ and $z$ of $P$ suç that $x$ and $y$ are independent modulo $\gamma_{2} P$ and $[x, y]=z^{m}$ for some $m>1$. Without loss of generality, we may assume that $m$ is a prime. Now, clearly, $z$ must lie in $\gamma_{2} P$. Furthermore, since $x$ and $y$ are independent modulo $\gamma_{2} P$, there exists a parabasis, consisting of elements $a_{1}$ and $b_{1}$ of $P$, such that

$$
\begin{aligned}
& y \equiv a_{1}^{r} \text { modulo } \gamma_{2} P, \\
& x \equiv a_{1}^{s} b_{1}^{k} \text { modulo } \gamma_{2} P .
\end{aligned}
$$


By a theorem of G. Baumslag [4], we may embed $P$ in a parafree metabelian group $\bar{P}$ so that $a_{1}$ has a $k$ th root $a$ in $\bar{P}$ and $a, b_{1}$ is a parabasis for $\bar{P}$. So in $\bar{P}$ we find

$$
\begin{aligned}
& y \equiv a^{r k} \text { modulo } \gamma_{2} \bar{P}, \\
& x \equiv a^{s k} b_{1}^{k}=\left(a^{s} b_{1}\right)^{k} \text { modulo } \gamma_{2} \bar{P} .
\end{aligned}
$$

Moreover the elements $a$ and $b=\dot{a}^{s} b_{1}$ form a parabasis for $\bar{P}$ and we have, setting $l=r k$,

$$
\begin{aligned}
& y \equiv a^{l} \text { modulo } \gamma_{2} \bar{P}, \\
& x \equiv b^{k} \text { modulo } \gamma_{2} \bar{P},
\end{aligned}
$$

with $r, s, t$ positive integers. Thus it follows from the discussion in $\$ 2$ of Chapter I that $x$ and $y$ can be written in the following way:

$$
y=a^{l} \prod_{m>0 ; n \geq 0}[b, m a, n b]^{l_{m, n}},
$$

and

$$
x=b^{k} \prod_{m>0 ; n \geq 0}[b, m a, n b]^{k} m, n .
$$

Using Lemma 2.3 of Chapter II we get

$$
\begin{aligned}
{[x, y]=} & {\left[b^{k}, a^{l}\right] \prod_{m>0 ; n \geq 0}\left([b, m a, n b]^{b^{k}}\right)^{-l_{m, n}} \cdot \prod_{m>0 ; n \geq 0}\left([b, m a, n b]^{a l}\right)^{k} m, n } \\
& \cdot \prod_{m>0 ; n \geq 0}[b, m a, n b]^{l_{m, n}} \prod_{m>0 ; n \geq 0}[b, m a, n b]^{-k_{m}, n} .
\end{aligned}
$$

Now, it follows from equation (5) and Lemmas 3.6 and 3.7 of Chapter I and 2.2 of Chapter II that

$$
\begin{aligned}
{[x, y]=} & \prod_{0 \leq s \leq k-1 ; 0 \leq t \leq l-1} \prod_{j=0}^{s}\left(\prod_{i=0}^{t}[b,(1+i) a, j b]^{m(i, t)}\right)^{m(j, s)} \\
& \cdot \prod_{m>0 ; n \geq 0}\left(\prod_{i=0}^{k}[b, m a,(n+i) b]^{m(i, k)}\right)^{-l} m, n \\
& \cdot \prod_{m>0 ; n \geq 0}\left(\prod_{i=0}^{l}[b,(m+i) a, n b]^{m(i, l)}\right)^{k, n} \\
& \cdot \prod_{m>0 ; n \geq 0}[b, m a, n b]^{l_{m, n}} \prod_{m>0 ; n \geq 0}[b, m a, n b]^{-k_{m, n}}
\end{aligned}
$$

Since we are assuming that $[x, y]=z^{m}$ where $m$ is a positive prime, the expo- 
nent of each commutator $[b, m a, n b]$ on the left side of equation (6) must divide $m$. However, we shall show that this is not the case.

Define a positive integer $\gamma$ as follows: If $m \nmid m(1, l)$, put $\gamma=1$. If $m \mid m(1, l)$, let $\gamma$ be the least positive integer such that $m \nmid m(\gamma, l)$. Since $m(l, l)=1$, it follows that $\gamma$ exists and $1 \leq \gamma \leq l$.

Similarly, define a positive integer $\delta$ as follows: If $m \nmid m(1, k)$, put $\delta=0$. If $m \mid m(1, k)$, let $\delta+1$ be the least positive integer such that $m \nmid m(\delta+1, k)$. Again, since $m(k, k)=1$, it follows that $\delta$ exists and $0 \leq \delta \leq k-1$.

Now, we shall show that the exponent $\epsilon$ of the commutator $[b, \gamma a, \delta b]$ on the left-hand side of equation (6) does not divide $m$. Using equation (6) and the definition of $m(s, t)$ we get

$$
\begin{aligned}
\epsilon= & \sum_{\substack{0 \leq t \leq k-1 ; 0 \leq s \leq l-1 \\
\gamma-1}} m(\gamma-1, t) m(\delta, s)-\sum_{i=0}^{\delta} l_{\gamma, \delta-i} m(i, k) \\
& +\sum_{\substack{l=0 \\
k-i, \delta}} m(i, l)+l_{\gamma, \delta}-k_{\gamma, \delta} \\
= & \sum_{t=0}^{k-1} m(\gamma-1, t) \sum_{s=0}^{l-1} m(\delta, s)-\sum_{i=1}^{\delta} l_{\gamma, \delta-i} m(i, k)+\sum_{i=1}^{\gamma-1} k_{\gamma-i, \delta} m(i, l) \\
= & m(\gamma, k) m(\delta+1, l)-\sum_{i=1}^{\delta} l_{\gamma, \delta-i} m(i, k)+\sum_{i=1}^{\gamma-1} k_{\gamma-i, \delta} m(i, l) .
\end{aligned}
$$

Now by the choice of $\gamma$ and $\delta$ we know that $m$ does not divide $m(\gamma, k)_{m}(\delta+1, l)$ but $m$ does divide every other term on the left side of equation (7). Thus $m$ does not divide $\epsilon$ and the lemma is proved.

\section{BIBLIOGRAPHY}

1. M. Auslander and R. C. Lyndon, Commutator subgroups of free groups, Amer. J. Math. 77 (1955), 929-931. MR 17, 709.

2. G. Baumslag, Some aspects of groups with unique roots, Acta Math. 104 (1960), 217-303. MR 23 \#A191.

3. - Some theorems on the free groups of certain product varieties, J. Combinatorial Theory 2 (1967), 77-99. MR 34 \#5902.

4. - Groups with the same lower central sequence as a relatively free group.

I. The groups, Trans. Amer. Math. Soc. 129 (1967), 308-321. MR 36 \#248.

5. - Groups with the same lower central sequence as a relatively free group.

II. Properties, Trans. Amer. Math. Soc. 142 (1969), 507-538. MR $39 \# 6959$.

6. G. Baumslag, B. H. Neumann, H. Neumann, and P. M. Neumann, On varieties generated by a finitely generated group, Math. Z. 86 (1964), 93-122. MR 30 \#138.

7. P. Hall, Some word-problems, J. Lond on Math. Soc. 33 (1958), 482-496. MR $21 \# 1331$.

8. A. Karrass, W. Magnus and D. Solitar, Elements of finite order in groups with a single defining relation, Comm. Pure Appl. Math. 13 (1960), 57-66. MR 23 \#A1696.

9. H. Neumann, Varieties of groups, Ergebnisse der Mathe matik und ihrer Grenzgebiete, Band 37, Springer-Verlag, New York, 1967. MR 35 \#6734. 
10. O. Schreier, Die Untergruppen der freien Gruppen, Abh. Math. Sem. Univ. Hamburg 5 (1928), 161-183.

11. J.-P. Serre, Lie algebras and Lie groups, Benjamin, New York, 1965. MR $36 \# 1582$.

DEPARTMENT OF MATHEMATICS, CITY UNIVERSITY OF NEW YORK, GRADUATE CENTER, NEW YORK, NEW YORK 10036

DEP ARTMENT OF MATHEM ATICS, NEW YORK INSTITUTE OF TECHNOLOGY, OLD WESTBURY, L. I., NEW YORK 11568 